\title{
DYNAMICAL TENSOR APPROXIMATION
}

\author{
OTHMAR KOCH* AND CHRISTIAN LUBICH ${ }^{\ddagger}$
}

\begin{abstract}
For the approximation of time-dependent data tensors and of solutions to tensor differential equations by tensors of low Tucker rank, we study a computational approach that can be viewed as a continuous-time updating procedure. This approach works with the increments rather than the full tensor and avoids the computation of decompositions of large matrices. In this method, the derivative is projected onto the tangent space of the manifold of tensors of Tucker rank $\left(r_{1}, \ldots, r_{N}\right)$ at the current approximation. This yields nonlinear differential equations for the factors in a Tucker decomposition, suitable for numerical integration. Approximation properties of this approach are analyzed.
\end{abstract}

Key words. Low-rank approximation, time-varying tensors, continuous updating, Tucker decomposition, tensor differential equations.

AMS subject classifications. 15A18,15A69,65F99,65L05

1. Introduction. Tensor approximation is an active research area with both interesting theoretical questions and numerous applications in the compression and retrieval of large structured data; see, e.g., $[3,4,6,11,19]$ and references therein. While these papers deal with the approximation of a fixed tensor, we here consider the approximation of time-varying (or parameter-dependent) tensors by tensors of low Tucker-rank. There arises the question as how to update a given tensor approximation, a problem that has been addressed in a different, discrete-time setting in $[16,17]$. The approach considered in the present paper can be viewed as a continuous-time updating technique, which works only with the increments in the tensors rather than the tensors themselves and does not require to compute any decompositions of large matrices. It extends the dynamical low-rank approximation of matrices $[8,15]$ to the higher-order tensor case.

Consider a time-varying family of tensors $\mathcal{A}(t) \in \mathbb{R}^{I_{1} \times \ldots \times I_{N}}$, for $0 \leq t \leq \bar{t}$. Let $\mathcal{M}_{\mathbf{r}}$ denote the manifold of all order- $N$ tensors of Tucker rank $\mathbf{r}=\left(r_{1}, \ldots, r_{N}\right)$ (see $\S 2$ below for the notion of Tucker rank or mode rank), where $r_{n} \leq I_{n}$ (and typically $r_{n} \ll I_{n}$ ) for $n=1, \ldots, N$. The best approximation to $\mathcal{A}(t)$ in $\overline{\mathcal{M}}_{\mathbf{r}}$ (with respect to the Frobenius norm $\|\cdot\|)$ is

$$
\boldsymbol{X}(t) \in \mathcal{M}_{\mathbf{r}} \quad \text { such that } \quad\|\boldsymbol{X}(t)-\mathcal{A}(t)\|=\min .
$$

Here, we consider instead the dynamical tensor approximation $\mathbf{y}(t) \in \mathcal{M}_{\mathbf{r}}$ determined from the condition that for every $t$ the derivative $\dot{\boldsymbol{y}}(t)$, which is in the tangent space $\mathcal{T}_{\mathbf{y}(t)} \mathcal{M}_{\mathbf{r}}$, be chosen as

$$
\dot{\mathbf{y}}(t) \in \mathcal{T}_{\mathbf{y}(t)} \mathcal{M}_{\mathbf{r}} \quad \text { such that } \quad\|\dot{\boldsymbol{y}}(t)-\dot{\mathcal{A}}(t)\|=\min .
$$

This is complemented with an initial condition, ideally $\boldsymbol{y}(0)=\boldsymbol{X}(0)$. Note that for given $\boldsymbol{y}(t)$, the derivative $\dot{\boldsymbol{y}}(t)$ is obtained in (1.2) by a linear projection, though onto a state-dependent vector space. Problem (1.2) yields an initial value problem of nonlinear ordinary differential equations on $\mathcal{M}_{\mathbf{r}}$, which becomes numerically efficiently

\footnotetext{
*Vienna University of Technology, Institute for Analysis and Scientific Computing, Wiedner Hauptstrasse 8-10, A-1040 Wien, Austria. Email: othmar@othmar-koch.org

${ }^{\ddagger}$ Mathematisches Institut, Universität Tübingen, Auf der Morgenstelle 10, D-72076 Tübingen, Germany. Email: lubich@na.uni-tuebingen.de
} 
accessible as differential equations for the factors in the Tucker decomposition of tensors of Tucker rank $\left(r_{1}, \ldots, r_{N}\right)$.

In a different context, a closely related approach was developed in the multiconfiguration time-dependent Hartree method of multi-particle quantum dynamics [2, 14], where the multidimensional time-dependent wave function is approximated by a linear combination of products of functions depending only on a single spatial variable.

We will study theoretical properties of the dynamical tensor approximation (1.2) in this paper: in $\S 2$ we derive the differential equations that are to be solved numerically, and after an auxiliary section on the tangent space projection ( $§ 3$ ) we study approximation properties in $\S 4$. Some numerical experiments are given in $\S 5$ (see also [15] for further numerical experiments with time-dependent 3-tensors that arise from a discretized reaction-diffusion PDE in 3 space dimensions).

The present paper extends the dynamical low-rank approximation of matrices, studied in our paper [8], to tensors. Though there are many conceptual similarities with the matrix case, the analysis of the tensor case is not simply a straightforward extension and therefore requires a careful discussion. While we have kept the general organisation of the paper largely parallel to [8] to make similarities and differences easily visible, we note that the material in $\S 2$ is quite apart from the matrix case in both notation and techniques, and the analysis of the tangent space projection in $\S 3$ requires different arguments. Once the projection estimates from $\S 3$ are available, some of the results in Section 4 then have essentially the same proof as the corresponding results in the matrix case (Theorems 4.1 and 4.2), whereas the proof of Theorem 4.3 follows a different line.

\section{Differential equations for dynamical tensor approximation.}

2.1. Prerequisites. We use the tensor notation of the review article [9], to which we refer for further details, results and numerous references.

Norm and inner product of tensors. The norm of a tensor $\boldsymbol{y} \in \mathbb{R}^{I_{1} \times \cdots \times I_{N}}$ is the Euclidean norm of the vector $\mathbf{y}$ that carries the entries $y_{i_{1}, \ldots, i_{N}}$ of $\boldsymbol{y}$. The inner product $\langle\boldsymbol{X}, \boldsymbol{y}\rangle$ of two tensors $\boldsymbol{X}, \boldsymbol{y} \in \mathbb{R}^{I_{1} \times \cdots \times I_{N}}$ is the Euclidean inner product of the two corresponding vectors $\mathbf{x}$ and $\mathbf{y}$.

Unfolding and reconstruction. The $n$th unfolding of a tensor $\boldsymbol{y} \in \mathbb{R}^{I_{1} \times \cdots \times I_{N}}$ is the matrix $\mathbf{Y}_{(n)} \in \mathbb{R}^{I_{n} \times I_{n+1} \ldots I_{N} I_{1} \ldots I_{n-1}}$ that aligns all entries $y_{i_{1}, \ldots, i_{N}}$ with fixed $i_{n}$ in the $i_{n}$-th row of the matrix, ordered lexicographically. We denote

$$
\mathbf{Y}_{(n)}=[\mathbf{y}]_{(n)},
$$

and clearly the tensor $\boldsymbol{y}$ can be reshaped from its unfolding $\mathbf{Y}_{(n)}$ : we write

$$
\boldsymbol{y}=\left[\mathbf{Y}_{(n)}\right]^{(n)} .
$$

The n-mode product. For a tensor $\boldsymbol{y} \in \mathbb{R}^{I_{1} \times \cdots \times I_{N}}$ and a matrix $\mathbf{V} \in \mathbb{R}^{J_{n} \times I_{n}}$, the $n$-mode product

$$
\boldsymbol{y} \times_{n} \mathbf{V} \in \mathbb{R}^{I_{1} \times \ldots \times I_{n-1} \times J_{n} \times I_{n+1} \times \ldots \times I_{N}}
$$

is defined by the relation

$$
\left[\mathbf{y} \times_{n} \mathbf{V}\right]_{(n)}=\mathbf{V} \mathbf{Y}_{(n)} .
$$

In particular, we then have, for another matrix $\mathbf{W}$ of appropriate dimension,

$$
\left(\boldsymbol{y} \times_{n} \mathbf{V}\right) \times_{n} \mathbf{W}=\boldsymbol{y} \times_{n}(\mathbf{W V}) .
$$


2.2. Manifold of rank- $\left(r_{1}, \ldots, r_{N}\right)$ tensors and Tucker decomposition. The $n$-rank of a tensor $\boldsymbol{y} \in \mathbb{R}^{I_{1} \times \cdots \times I_{N}}$ is

$$
r_{n}=\operatorname{rank}\left(\mathbf{Y}_{(n)}\right) \text {, }
$$

and the vector $\mathbf{r}=\left(r_{1}, \ldots, r_{N}\right)$ is known as the Tucker rank of the tensor. For given $n$-rank $r_{n} \leq I_{n}$ (and typically $r_{n} \ll I_{n}$ ), the set

$$
\mathcal{M}_{\mathbf{r}}=\left\{\boldsymbol{y} \in \mathbb{R}^{I_{1} \times \cdots \times I_{N}}: \mathbf{y} \text { has } n \text {-rank } r_{n} \text { for } n=1, \ldots, N\right\}
$$

is a manifold that will serve as an approximation manifold for general tensors $\mathcal{A} \in$ $\mathbb{R}^{I_{1} \times \cdots \times I_{N}}$. As is known (see [10]), every tensor $\boldsymbol{y} \in \mathcal{M}_{\mathbf{r}}$ can be written as a Tucker decomposition

$$
\boldsymbol{y}=\mathfrak{S} \times{ }_{1} \mathbf{U}_{1} \ldots \times_{N} \mathbf{U}_{N}=: \mathfrak{S} \stackrel{N}{\underset{X}{X}} \mathbf{U}_{n}
$$

where the core tensor $\mathcal{S} \in \mathbb{R}^{r_{1} \times \cdots \times r_{N}}$ is of full Tucker rank $\mathbf{r}=\left(r_{1}, \ldots, r_{N}\right)$, and the matrices $\mathbf{U}_{n} \in \mathbb{R}^{I_{n} \times r_{n}}$ have orthonormal columns $\mathbf{u}_{j_{n}}^{(n)}\left(j_{n}=1, \ldots, r_{n}\right)$. In terms of the entries of $\mathcal{S}=\left(s_{j_{1} \ldots j_{N}}\right)$, the above expression can be rewritten as a linear combination of rank-1 tensors that are formed as the outer products of the column vectors:

$$
\boldsymbol{y}=\sum_{j_{1}, \ldots, j_{N}} s_{j_{1} \ldots j_{N}} \mathbf{u}_{j_{1}}^{(1)} \circ \ldots \circ \mathbf{u}_{j_{N}}^{(N)}
$$

In terms of the $n$th unfolding, we have the useful matrix formula

$$
\mathbf{Y}_{(n)}=\mathbf{U}_{n} \mathbf{S}_{(n)}\left(\mathbf{U}_{N} \otimes \ldots \otimes \mathbf{U}_{n+1} \otimes \mathbf{U}_{n-1} \otimes \ldots \otimes \mathbf{U}_{1}\right)^{T}=: \mathbf{U}_{n} \mathbf{S}_{(n)} \bigotimes_{k \neq n} \mathbf{U}_{k}^{T},
$$

where $\otimes$ denotes the Kronecker matrix product.

The representation (2.3) is not unique: replacing $\mathbf{U}_{n}$ by $\tilde{\mathbf{U}}_{n}=\mathbf{U}_{n} \mathbf{Q}_{n}$ with orthogonal matrices $\mathbf{Q}_{n}$ and $\mathcal{S}$ by $\tilde{\mathcal{S}}=\mathcal{S} \mathbf{X}_{n=1}^{N} \mathbf{Q}_{n}^{T}$, yields the same tensor $\boldsymbol{y}=\boldsymbol{S} \mathbf{X}_{n=1}^{N} \mathbf{U}_{n}=$ $\tilde{\mathbf{S}} \mathbf{X}_{n=1}^{N} \tilde{\mathbf{U}}_{n}$.

2.3. Tangent tensors. As a substitute for the non-uniqueness in the decomposition (2.3), we will use a unique decomposition in the tangent space. Let $\mathcal{V}_{I, r}$ denote the Stiefel manifold of real $I \times r$ matrices with orthonormal columns. The tangent space at $\mathbf{U} \in \mathcal{V}_{I, r}$ is

$$
\mathcal{T}_{\mathbf{U}} \mathcal{V}_{I, r}=\left\{\dot{\mathbf{U}} \in \mathbb{R}^{I \times r}: \dot{\mathbf{U}}^{T} \mathbf{U}+\mathbf{U}^{T} \dot{\mathbf{U}}=\mathbf{0}\right\}=\left\{\dot{\mathbf{U}} \in \mathbb{R}^{I \times r}: \mathbf{U}^{T} \dot{\mathbf{U}} \in \operatorname{so}(r)\right\},
$$

where so $(r)$ denotes the space of skew-symmetric real $r \times r$ matrices. Consider the extended tangent map of $\left(\mathcal{S}, \mathbf{U}_{1}, \ldots, \mathbf{U}_{N}\right) \mapsto \boldsymbol{y}=\boldsymbol{S} \mathbf{X}_{n=1}^{N} \mathbf{U}_{n}$,

$$
\begin{aligned}
& \mathbb{R}^{r_{1} \times \ldots \times r_{N}} \times \prod_{n=1}^{N} \mathcal{T}_{\mathbf{U}_{n}} \mathcal{V}_{I_{n}, r_{n}} \rightarrow \mathcal{T}_{\boldsymbol{y}} \mathcal{M}_{\mathbf{r}} \times \prod_{n=1}^{N} \operatorname{so}\left(r_{n}\right) \\
& \left(\dot{\mathcal{S}}, \dot{\mathbf{U}}_{1}, \ldots, \dot{\mathbf{U}}_{N}\right) \mapsto\left(\dot{\mathcal{S}} \underset{n=1}{\mathbf{X}} \mathbf{U}_{n}+\sum_{n=1}^{N} \boldsymbol{S} \times_{n} \dot{\mathbf{U}}_{n} \underset{k \neq n}{\mathbf{X}} \mathbf{U}_{k}, \mathbf{U}_{1}^{T} \dot{\mathbf{U}}_{1}, \ldots, \mathbf{U}_{N}^{T} \dot{\mathbf{U}}_{N}\right) .
\end{aligned}
$$


This linear map turns out to be an isomorphism, since its inverse can be constructed explicitly by an argument similar to the proof of (2.7) below. Hence, every tangent tensor $\dot{y} \in \mathcal{T}_{\mathbf{y}} \mathcal{M}_{\mathbf{r}}$ is of the form

$$
\dot{\boldsymbol{y}}=\dot{\mathcal{S}} \underset{n=1}{\mathrm{X}} \mathbf{U}_{n}+\sum_{n=1}^{N} \boldsymbol{S} \times_{n} \dot{\mathbf{U}}_{n} \underset{k \neq n}{\mathrm{X}} \mathbf{U}_{k},
$$

where $\dot{\mathcal{S}} \in \mathbb{R}^{r_{1} \times \ldots \times r_{N}}$ and $\dot{\mathbf{U}}_{n} \in \mathcal{T}_{\mathbf{U}_{n}} \mathcal{V}_{I_{n}, r_{n}}$. Moreover, it follows that $\dot{\mathcal{S}}$ and $\dot{\mathbf{U}}_{n}$ are uniquely determined by $\dot{\boldsymbol{y}}$ and the chosen $\boldsymbol{S}$ and $\mathbf{U}_{n}$ in (2.3) if we impose the orthogonality constraints

$$
\mathbf{U}_{n}^{T} \dot{\mathbf{U}}_{n}=\mathbf{0}
$$

We now show that then $\dot{\mathcal{S}}$ and $\dot{\mathbf{U}}_{n}$ are actually given by the following formulas:

$$
\begin{aligned}
\dot{\boldsymbol{S}} & =\dot{\boldsymbol{y}} \underset{n=1}{\mathrm{X}} \mathbf{U}_{n}^{T} \\
\dot{\mathbf{U}}_{n} & =\mathbf{P}_{n}^{\perp}\left[\dot{\boldsymbol{y}} \underset{k \neq n}{\mathbf{X}} \mathbf{U}_{k}^{T}\right]_{(n)} \mathbf{S}_{(n)}^{\dagger}
\end{aligned}
$$

with the projection $\mathbf{P}_{n}^{\perp}=\mathbf{I}-\mathbf{U}_{n} \mathbf{U}_{n}^{T}$ and the pseudo-inverse $\mathbf{S}_{(n)}^{\dagger}=\mathbf{S}_{(n)}^{T}\left(\mathbf{S}_{(n)} \mathbf{S}_{(n)}^{T}\right)^{-1}$. The $r_{n} \times r_{n}$ matrix $\mathbf{S}_{(n)} \mathbf{S}_{(n)}^{T}$ is invertible since $\mathbf{S}_{(n)}$ is of rank $r_{n}$ by assumption.

Proof of (2.7): When we multiply (2.5) by $\mathbf{X}_{n=1}^{N} \mathbf{U}_{n}^{T}$, then we obtain by $(2.2)$ and the orthogonality relations $\mathbf{U}_{n}^{T} \mathbf{U}_{n}=\mathbf{I}$ and (2.6) the first equation of (2.7). For the second equation, we multiply $(2.5)$ by $\mathbf{X}_{k \neq n} \mathbf{U}_{k}^{T}$, and again using (2.2) and the orthogonality relations we obtain

$$
\dot{\mathrm{y}} \underset{k \neq n}{\mathrm{X}} \mathbf{U}_{k}^{T}=\dot{\mathfrak{S}} \times_{n} \mathbf{U}_{n}+\mathcal{S} \times_{n} \dot{\mathbf{U}}_{n}
$$

By the equation for $\dot{\mathcal{S}}$ and once again (2.2), the first expression on the right-hand side becomes

$$
\dot{\mathcal{S}} \times{ }_{n} \mathbf{U}_{n}=\dot{\boldsymbol{y}} \times{ }_{n}\left(\mathbf{U}_{n} \mathbf{U}_{n}^{T}\right) \underset{k \neq n}{X} \mathbf{U}_{k}^{T},
$$

or in its mode- $n$ unfolding,

$$
\mathbf{U}_{n} \dot{\mathbf{S}}_{(n)}=\mathbf{U}_{n} \mathbf{U}_{n}^{T}\left[\dot{\boldsymbol{y}} \underset{k \neq n}{\mathbf{X}} \mathbf{U}_{k}^{T}\right]_{(n)} .
$$

Taking the mode- $n$ unfolding on both sides of (2.8) and rearranging terms then gives

$$
\dot{\mathbf{U}}_{n} \mathbf{S}_{(n)}=\left(\mathbf{I}-\mathbf{U}_{n} \mathbf{U}_{n}^{T}\right)\left[\dot{\boldsymbol{y}} \underset{k \neq n}{\mathbf{X}} \mathbf{U}_{k}^{T}\right]_{(n)} .
$$

Multiplying this relation from the right with $\mathbf{S}_{(n)}^{\dagger}$ yields the second equation of (2.7).

2.4. Dynamical tensor approximation. We now turn to the approximation (1.2) of time-dependent tensors $\mathcal{A}(t)$. The minimization condition (1.2) on the tangent space is equivalent to an orthogonal projection: find $\dot{\boldsymbol{y}} \in \mathcal{T}_{\mathbf{y}} \mathcal{M}_{\mathbf{r}}$ (we omit the argument $t$ ) satisfying

$$
\langle\dot{\boldsymbol{y}}-\dot{\mathcal{A}}, \mathcal{V}\rangle=0 \quad \text { for all } \mathcal{V} \in \mathcal{T}_{\boldsymbol{y}} \mathcal{M}_{\mathbf{r}}
$$


This is a Galerkin condition on the tangent space $\mathcal{T}_{\boldsymbol{y}} \mathcal{M}_{\mathbf{r}}$. With this formulation we derive differential equations for the factors in the Tucker decomposition (2.3).

THEOREM 2.1. For a tensor $\boldsymbol{y}=\mathbf{S}_{n=1}^{N} \mathbf{U}_{n} \in \mathcal{M}_{\mathbf{r}}$ with n-rank $r_{n}$ for $n=$ $1, \ldots, N$, with core tensor $\mathcal{S} \in \mathbb{R}^{r_{1} \times \cdots \times r_{N}}$ and n-mode factors $\mathbf{U}_{n} \in \mathbb{R}^{I_{n} \times r_{n}}$ having orthonormal columns, condition (1.2) or (2.9) is equivalent to

$$
\dot{\boldsymbol{y}}=\dot{\mathcal{S}} \underset{n=1}{\mathrm{X}} \mathbf{U}_{n}+\sum_{n=1}^{N} \mathcal{S} \times_{n} \dot{\mathbf{U}}_{n} \underset{k \neq n}{\mathbf{X}} \mathbf{U}_{k},
$$

where the factors in the decomposition satisfy the system of differential equations

$$
\begin{aligned}
\dot{\mathcal{S}} & =\dot{\mathcal{A}} \underset{n=1}{\stackrel{N}{X} \mathbf{U}_{n}^{T}} \\
\dot{\mathbf{U}}_{n} & =\mathbf{P}_{n}^{\perp}\left[\dot{\mathcal{A}} \underset{k \neq n}{\mathbf{X}} \mathbf{U}_{k}^{T}\right]_{(n)} \mathbf{S}_{(n)}^{\dagger}
\end{aligned}
$$

with the projection $\mathbf{P}_{n}^{\perp}=\mathbf{I}-\mathbf{U}_{n} \mathbf{U}_{n}^{T}$ onto the orthogonal complement of the range of $\mathbf{U}_{n}$ and with the pseudo-inverse $\mathbf{S}_{(n)}^{\dagger}=\mathbf{S}_{(n)}^{T}\left(\mathbf{S}_{(n)} \mathbf{S}_{(n)}^{T}\right)^{-1}$ of the n-mode unfolding $\mathbf{S}_{(n)}$ of $\mathcal{S}$.

Equations (2.11) are formally like (2.7), with $\dot{\boldsymbol{y}}$ replaced by $\dot{\mathcal{A}}$.

The differential equations (2.11) are solved numerically, starting from an approximation to the tensor $\mathcal{A}(0)$ at the initial time given in the low-rank Tucker format. Notice that a time step with these differential equations requires no decompositions of large matrices (the matrices $\mathbf{S}_{(n)} \mathbf{S}_{(n)}^{T}$ are of small dimension $r_{n} \times r_{n}$ ). The main computational work is in the computation of the dimension-contracting $n$-mode products appearing on the right-hand side of (2.11). These require inner products of length $I_{n}$ and can exploit possible sparsity in $\dot{\mathcal{A}}(t)$ so that only the actually time-varying entries of $\mathcal{A}(t)$ are addressed.

Proof. We know from Section 2.3 that $\dot{\boldsymbol{y}}$ can be written in the form (2.10) with $\dot{\mathbf{U}}_{n}$ satisfying the orthogonality condition (2.6). Choosing $\mathcal{V}=\mathcal{T} \mathbf{X}_{n=1}^{N} \mathbf{U}_{n} \in \mathcal{T}_{\mathbf{y}} \mathcal{M}_{\mathbf{r}}$ with an arbitrary $\mathcal{T} \in \mathbb{R}^{r_{1} \times \cdots \times r_{N}}$, we have

$$
\begin{aligned}
& \left\langle\dot{\mathcal{A}}, \mathcal{T} \stackrel{N=1}{X}_{n}^{N} \mathbf{U}_{n}\right\rangle=\left\langle\dot{\mathcal{A}} \underset{n=1}{\stackrel{N}{X}} \mathbf{U}_{n}^{T}, \mathcal{T}\right\rangle \\
& \left\langle\dot{\boldsymbol{y}}, \mathcal{T} \underset{n=1}{N} \mathbf{U}_{n}\right\rangle=\langle\dot{\mathcal{S}}, \mathfrak{T}\rangle .
\end{aligned}
$$

Since this holds for every $\mathcal{T} \in \mathbb{R}^{r_{1} \times \cdots \times r_{N}},(2.9)$ yields the first equation of (2.11).

We now choose $\mathcal{V}=\boldsymbol{S} \times{ }_{n} \mathbf{V}_{n} \mathbf{X}_{k \neq n} \mathbf{U}_{k}$ which is in $\mathcal{T}_{\boldsymbol{y}} \mathcal{M}_{\mathbf{r}}$ if $\mathbf{V}_{n}$ satisfies the orthogonality relation $\mathbf{U}_{n}^{T} \mathbf{V}_{n}=\mathbf{0}$. We then have

$$
\begin{aligned}
\langle\dot{\mathcal{A}}, & \left.\boldsymbol{S} \times_{n} \mathbf{V}_{n} \underset{k \neq n}{\mathbf{X}} \mathbf{U}_{k}\right\rangle=\left\langle\dot{\mathcal{A}} \underset{k \neq n}{\mathbf{X}} \mathbf{U}_{k}^{T}, \boldsymbol{S} \times_{n} \mathbf{V}_{n}\right\rangle \\
& =\left\langle\left[\dot{\mathcal{A}} \underset{k \neq n}{\mathbf{X}} \mathbf{U}_{k}^{T}\right]_{(n)}, \mathbf{V}_{n} \mathbf{S}_{(n)}\right\rangle=\left\langle\left[\dot{\mathcal{A}} \underset{k \neq n}{\mathbf{X}} \mathbf{U}_{k}^{T}\right]_{(n)} \mathbf{S}_{(n)}^{T}, \mathbf{V}_{n}\right\rangle,
\end{aligned}
$$

where the matrix inner product in the second line is the Frobenius inner product. On 
the other hand we have, using (2.10) and the orthogonality relations,

$$
\begin{aligned}
\left\langle\dot{\boldsymbol{y}}, \boldsymbol{S} \times_{n} \mathbf{V}_{n} \underset{k \neq n}{\mathbf{X}} \mathbf{U}_{k}\right\rangle & =\left\langle\dot{\mathcal{S}} \times_{n} \mathbf{U}_{n}+\mathcal{S} \times_{n} \dot{\mathbf{U}}_{n}, \boldsymbol{S} \times_{n} \mathbf{V}_{n}\right\rangle \\
& =\left\langle\mathbf{U}_{n} \dot{\mathbf{S}}_{(n)}+\dot{\mathbf{U}}_{n} \mathbf{S}_{(n)}, \mathbf{V}_{n} \mathbf{S}_{(n)}\right\rangle \\
& =\left\langle\dot{\mathbf{S}}_{(n)}, \mathbf{U}_{n}^{T} \mathbf{V}_{n} \mathbf{S}_{(n)}\right\rangle+\left\langle\dot{\mathbf{U}}_{n} \mathbf{S}_{(n)} \mathbf{S}_{(n)}^{T}, \mathbf{V}_{n}\right\rangle \\
& =\left\langle\dot{\mathbf{U}}_{n} \mathbf{S}_{(n)} \mathbf{S}_{(n)}^{T}, \mathbf{V}_{n}\right\rangle .
\end{aligned}
$$

By (2.9), we thus have

$$
\left\langle\dot{\mathbf{U}}_{n} \mathbf{S}_{(n)} \mathbf{S}_{(n)}^{T}-\left[\dot{\mathcal{A}} \underset{k \neq n}{\mathbf{X}} \mathbf{U}_{k}^{T}\right]_{(n)} \mathbf{S}_{(n)}^{T}, \mathbf{V}_{n}\right\rangle=0
$$

for all $\mathbf{V}_{n}$ with $\mathbf{U}_{n}^{T} \mathbf{V}_{n}=\mathbf{0}$, that is, for all matrices $\mathbf{V}_{n}=\left(\mathbf{I}-\mathbf{U}_{n} \mathbf{U}_{n}^{T}\right) \mathbf{W}_{n}$ with an arbitrary matrix $\mathbf{W}_{n} \in \mathbb{R}^{I_{n} \times r_{n}}$. We therefore obtain

$$
\left(\mathbf{I}-\mathbf{U}_{n} \mathbf{U}_{n}^{T}\right) \dot{\mathbf{U}}_{n} \mathbf{S}_{(n)} \mathbf{S}_{(n)}^{T}=\left(\mathbf{I}-\mathbf{U}_{n} \mathbf{U}_{n}^{T}\right)\left[\dot{\mathcal{A}} \underset{k \neq n}{\mathbf{X}} \mathbf{U}_{k}^{T}\right]_{(n)} \mathbf{S}_{(n)}^{T} .
$$

Since $\mathbf{U}_{n}^{T} \dot{\mathbf{U}}_{n}=\mathbf{0}$ by condition (2.6), this yields the second equation of (2.11).

REMARK 2.1. Related differential equations were derived earlier in the chemical physics literature in [2] for the multiconfiguration time-dependent Hartree method of quantum mechanics, where an approximation to the multivariate wave function is sought for in the form of a linear combination of products of univariate functions.

REMARK 2.2. In this paper we only deal with the Tucker format of tensor approximation. Another familiar format is the canonical format (for tensors of canonical $\operatorname{rank} K$ )

$$
\mathcal{A}(t) \approx \sum_{k=1}^{K} \mathbf{v}_{k}^{(1)}(t) \circ \ldots \circ \mathbf{v}_{k}^{(N)}(t)
$$

where no orthogonality relation is required between the vectors $\mathbf{v}_{k}^{(n)}(t)$. While this approach appears attractive in that it has no built-in exponential scaling with the order $N$ of the tensor, it leads to a number of theoretical difficulties even in the time-independent approximation problem [3] and does not appear to lend itself to the dynamical tensor approximation approach. The set of all tensors of canonical rank $K$ does not have a manifold structure that allows us to give differential equations for the vectors $\mathbf{v}_{k}^{(n)}(t)$ such that the analogue of (1.2) is fulfilled.

REMARK 2.3. The dynamical tensor approximation approach for the Tucker format can be extended to the hierarchical tensor format described in $[2,5,13,18]$, which has only cubic scaling with $N$. This is, however, outside the scope of the present paper.

3. Tangent space projection and curvature bounds. In operator notation, (2.9) can be written as

$$
\dot{\boldsymbol{y}}=\mathcal{P}(\boldsymbol{y}) \dot{\mathcal{A}}
$$

where $\mathcal{P}(\boldsymbol{y})$ is the orthogonal projection onto the tangent space $\mathcal{T}_{\boldsymbol{y}} \mathcal{M}_{\mathbf{r}}$. For a theoretical understanding of the dynamical low-rank approximation, an analysis of the properties of this projection operator is therefore essential. We begin by giving an explicit formula. 
Lemma 3.1. Let $\boldsymbol{y}=\boldsymbol{S} \mathbf{X}_{n=1}^{N} \mathbf{U}_{n} \in \mathcal{M}_{\mathbf{r}}$ with n-mode factors $\mathbf{U}_{n}$ having orthonormal columns. With the orthogonal projections

$$
\begin{aligned}
& \mathbf{P}_{n}=\mathbf{U}_{n} \mathbf{U}_{n}^{T}, \quad \mathbf{P}_{n}^{\perp}=\mathbf{I}-\mathbf{P}_{n}, \\
& \mathbf{Q}_{n}=\bigotimes_{k \neq n} \mathbf{U}_{k} \mathbf{S}_{(n)}^{\dagger} \mathbf{S}_{(n)} \bigotimes_{k \neq n} \mathbf{U}_{k}^{T},
\end{aligned}
$$

the orthogonal projection onto the tangent space $\mathcal{T}_{\mathbf{y}} \mathcal{M}_{\mathbf{r}}$ is given by

$$
\mathcal{P}(\boldsymbol{y}) \mathcal{B}=\mathcal{B} \underset{n=1}{\mathrm{X}} \mathbf{P}_{n}+\sum_{n=1}^{N}\left[\mathbf{P}_{n}^{\perp} \mathbf{B}_{(n)} \mathbf{Q}_{n}\right]^{(n)}
$$

for $\mathcal{B} \in \mathbb{R}^{I_{1} \times \cdots \times I_{N}}$, with $\mathbf{B}_{(n)}=[\mathcal{B}]_{(n)}$ the nth unfolding of $\mathcal{B}$.

Proof. Theorem 2.1 gives an expression for $\dot{\boldsymbol{y}}$ determined by (2.9), that is, for $\dot{\boldsymbol{y}}=\mathcal{P}(\boldsymbol{y}) \dot{\mathcal{A}}$. With $\mathcal{B}$ in place of $\dot{\mathcal{A}}$, this reads

$$
\begin{aligned}
& \mathcal{P}(\boldsymbol{y}) \mathcal{B}=\mathcal{B} \underset{n=1}{\stackrel{N}{X}} \mathbf{U}_{n}^{T} \underset{n=1}{\underset{X}{X}} \mathbf{U}_{n}+\sum_{n=1}^{N} \boldsymbol{S} \times_{n}\left(\mathbf{P}_{n}^{\perp}\left[\underset{k \neq n}{\boldsymbol{X}} \mathbf{U}_{k}^{T}\right]_{(n)} \mathbf{S}_{(n)}^{\dagger}\right) \underset{k \neq n}{\boldsymbol{X}} \mathbf{U}_{k} \\
& =\mathcal{B} \underset{n=1}{X} \mathbf{P}_{n}+\sum_{n=1}^{N}\left[\mathbf{P}_{n}^{\perp} \mathbf{B}_{(n)} \bigotimes_{k \neq n} \mathbf{U}_{k} \mathbf{S}_{(n)}^{\dagger} \mathbf{S}_{(n)} \bigotimes_{k \neq n} \mathbf{U}_{k}^{T}\right]^{(n)},
\end{aligned}
$$

where we have used (2.2) and (2.4). This is (3.2).

The next lemma is the key tool for the approximation results of the following section.

Lemma 3.2. There are constants $c$ and $C$ (depending only on the order $N$ and satisfying $\left.C c \leq \frac{1}{2}\right)$ such that the following holds true. Let the rank- $\left(r_{1}, \ldots, r_{N}\right)$ tensor $\boldsymbol{X} \in \mathcal{M}_{\mathbf{r}}$ be such that the smallest nonzero singular value of the nth unfolding satisfies $\sigma_{r_{n}}\left(\mathbf{X}_{(n)}\right) \geq \rho>0$ for $n=1, \ldots, N$, and let $\tilde{\boldsymbol{X}} \in \mathcal{M}_{\mathbf{r}}$ with $\|\tilde{\boldsymbol{X}}-\boldsymbol{X}\| \leq c \rho$. Then, for all $\mathbf{B} \in \mathbb{R}^{I_{1} \times \cdots \times I_{N}}$,

$$
\begin{gathered}
\|(\mathcal{P}(\tilde{\boldsymbol{X}})-\mathcal{P}(\boldsymbol{X})) \mathcal{B}\| \leq C \rho^{-1}\|\tilde{\boldsymbol{X}}-\boldsymbol{X}\| \cdot\|\mathcal{B}\|, \\
\left\|\mathcal{P}^{\perp}(\boldsymbol{X})(\tilde{\boldsymbol{x}}-\boldsymbol{X})\right\| \leq C \rho^{-1}\|\tilde{\boldsymbol{X}}-\boldsymbol{X}\|^{2}
\end{gathered}
$$

where $\mathcal{P}^{\perp}(\boldsymbol{X})=I-\mathcal{P}(\boldsymbol{X})$ is the projection onto the orthogonal complement of the tangent space $\mathcal{T}_{x} \mathcal{M}_{\mathbf{r}}$.

Proof. (a) Writing $\boldsymbol{X} \in \mathcal{M}_{\mathbf{r}}$ in Tucker form as $\boldsymbol{X}=\mathcal{S} \mathbf{X}_{n=1}^{N} \mathbf{U}_{n}$, we note that the non-zero singular values of $\mathbf{X}_{(n)}$ are those of $\mathbf{S}_{(n)}$, and hence

$$
\left\|\mathbf{S}_{(n)}^{\dagger}\right\|=\frac{1}{\sigma_{r_{n}}\left(\mathbf{S}_{(n)}\right)}=\frac{1}{\sigma_{r_{n}}\left(\mathbf{X}_{(n)}\right)} \leq \frac{1}{\rho} .
$$

Since we have, by [7, p. 448],

$$
\left|\sigma_{r_{n}}\left(\tilde{\mathbf{X}}_{(n)}\right)-\sigma_{r_{n}}\left(\mathbf{X}_{(n)}\right)\right| \leq\left\|\tilde{\mathbf{X}}_{(n)}-\mathbf{X}_{(n)}\right\|_{2} \leq\left\|\tilde{\mathbf{X}}_{(n)}-\mathbf{X}_{(n)}\right\|_{F}=\|\tilde{\boldsymbol{X}}-\boldsymbol{X}\|,
$$

we obtain for $\|\tilde{\boldsymbol{X}}-\boldsymbol{X}\| \leq \frac{1}{2} \rho$ that

$$
\sigma_{r_{n}}\left(\tilde{\mathbf{X}}_{(n)}\right) \geq \sigma_{r_{n}}\left(\mathbf{X}_{(n)}\right)-\left|\sigma_{r_{n}}\left(\tilde{\mathbf{X}}_{(n)}\right)-\sigma_{r_{n}}\left(\mathbf{X}_{(n)}\right)\right| \geq \frac{1}{2} \rho,
$$


and hence $\tilde{\boldsymbol{X}}=\tilde{\mathcal{S}} \mathbf{X}_{n=1}^{N} \tilde{\mathbf{U}}_{n}$ with $\left\|\tilde{\mathbf{S}}_{(n)}^{\dagger}\right\| \leq 2 \rho^{-1}$.

(b) We decompose the tensors on the straight line connecting $\boldsymbol{X}$ and $\tilde{\boldsymbol{X}}$ as

$$
\boldsymbol{X}+\tau(\tilde{\boldsymbol{X}}-\boldsymbol{X})=\boldsymbol{y}(\tau)+\boldsymbol{z}(\tau) \quad \text { with } \quad \boldsymbol{y}(\tau) \in \mathcal{M}_{\mathbf{r}}, \boldsymbol{z}(\tau) \perp \mathcal{T}_{\boldsymbol{X}} \mathcal{M}_{\mathbf{r}} .
$$

A smooth such decomposition exists at least for small $\tau$, but the arguments below show that it exists in fact for $0 \leq \tau \leq 1$. We denote

$$
\Delta=\mathcal{P}(\boldsymbol{X})(\tilde{\boldsymbol{X}}-\boldsymbol{X}) \in \mathcal{T}_{\boldsymbol{X}} \mathcal{M}_{\mathbf{r}}, \quad \text { with }\|\Delta\| \leq \delta:=\|\tilde{\boldsymbol{X}}-\boldsymbol{X}\| .
$$

We then have $\mathcal{P}(\boldsymbol{X})(\boldsymbol{y}(\tau)-\boldsymbol{X})=\tau \Delta$, which yields

$$
\mathcal{P}(\boldsymbol{X}) \dot{\boldsymbol{y}}(\tau)=\Delta .
$$

Since $\mathcal{P}(\boldsymbol{y}) \dot{\boldsymbol{y}}=\dot{\boldsymbol{y}}$, we have

$$
\dot{\boldsymbol{y}}(\tau)=\Delta+(\mathcal{P}(\boldsymbol{y}(\tau))-\mathcal{P}(\boldsymbol{X})) \dot{\boldsymbol{y}}(\tau) .
$$

(c) As long as the operator norm of $(\mathcal{P}(\boldsymbol{y}(\tau))-\mathcal{P}(\boldsymbol{X}))$ is bounded by $\frac{1}{2}$, we thus have

$$
\|\dot{\boldsymbol{y}}\| \leq 2 \delta .
$$

This yields $\|\boldsymbol{y}(\tau)-\boldsymbol{X}\| \leq 2 \delta$ for $0 \leq \tau \leq 1$, and for the Tucker factors of $\boldsymbol{y}(\tau)=$ $\boldsymbol{S}(\tau) \mathbf{X}_{n=1}^{N} \mathbf{U}_{n}(\tau)$ with $\mathbf{U}_{n}^{T} \dot{\mathbf{U}}_{n}=\mathbf{0}$ we therefore have by $(2.7)$

$$
\|\dot{\boldsymbol{\mathcal { S }}}\| \leq 2 \delta, \quad\left\|\dot{\mathbf{U}}_{n} \mathbf{S}_{(n)}\right\| \leq 2 \delta .
$$

If $2 \delta \leq \frac{1}{2} \rho$, then the argument of (a) applied to $\boldsymbol{y}(\tau)$ instead of $\tilde{\boldsymbol{X}}$ shows that $\left\|\mathbf{S}_{(n)}^{\dagger}(\tau)\right\| \leq 2 \rho^{-1}$, and hence

$$
\left\|\dot{\mathbf{U}}_{n}\right\| \leq\left\|\dot{\mathbf{U}}_{n} \mathbf{S}_{(n)}\right\| \cdot\left\|\mathbf{S}_{(n)}^{\dagger}\right\| \leq 4 \delta \rho^{-1}=: \gamma .
$$

With this estimate we further obtain for $\mathbf{P}_{n}=\mathbf{U}_{n} \mathbf{U}_{n}^{T}$ that

$$
\left\|\dot{\mathbf{P}}_{n}\right\| \leq 2 \gamma .
$$

Using the product rule for $\frac{d}{d \tau}\left(\mathbf{S}_{(n)}^{T}\left(\mathbf{S}_{(n)} \mathbf{S}_{(n)}^{T}\right)^{-1} \mathbf{S}_{(n)}\right)$ and the estimates for the norms of $\dot{\mathbf{S}}_{(n)}$ and $\mathbf{S}_{(n)}^{\dagger}$ by $2 \delta$ and $2 \rho^{-1}$, respectively, we find that the norm of this derivative is bounded by $4 \gamma$, and hence we have for the projection $\mathbf{Q}_{n}$ of Lemma 3.1

$$
\left\|\dot{\mathbf{Q}}_{n}\right\| \leq 2(N-1) \gamma+4 \gamma=2(N+1) \gamma .
$$

Expressing $\mathbf{U}_{n}(\tau)-\mathbf{U}_{n}(0)=\int_{0}^{\tau} \dot{\mathbf{U}}_{n}(s) d s$ and similarly for the increments of $\mathcal{S}, \mathbf{P}_{n}$, and $\mathbf{Q}_{n}$, we obtain from the formula of Lemma 3.1 and the above estimates

$$
\begin{gathered}
\|(\mathcal{P}(\mathbf{y}(\tau))-\mathcal{P}(\boldsymbol{X})) \mathcal{B}\| \leq(N \cdot 2 \gamma+N \cdot(2 \gamma+2(N+1) \gamma)) \tau\|\mathcal{B}\| \\
\quad=2 N(N+3) \gamma \cdot \tau\|\mathcal{B}\|=8 N(N+3) \delta \rho^{-1} \tau\|\mathcal{B}\| .
\end{gathered}
$$

The operator norm of $\mathcal{P}(\boldsymbol{y}(\tau))-\mathcal{P}(\boldsymbol{X})$ thus does not exceed $\frac{1}{2}$ for $0 \leq \tau \leq 1$ if

$$
\delta \leq c \rho \quad \text { with } c=\frac{1}{16 N(N+3)},
$$


and at $\tau=1$ we then obtain the bound (3.3) with $C=8 N(N+3)$.

(d) We note

$$
\mathcal{P}^{\perp}(\boldsymbol{X})(\tilde{\boldsymbol{X}}-\boldsymbol{X})=\mathcal{P}^{\perp}(\boldsymbol{X}) \int_{0}^{1} \dot{\boldsymbol{y}}(\tau) d \tau=\int_{0}^{1}(\mathcal{P}(\mathbf{y}(\tau))-\mathcal{P}(\boldsymbol{X})) \dot{\boldsymbol{y}}(\tau) d \tau .
$$

By the above estimates, this is bounded by

$$
\left\|\mathcal{P}^{\perp}(\boldsymbol{X})(\tilde{\boldsymbol{X}}-\boldsymbol{X})\right\| \leq 8 N(N+3) \rho^{-1} \delta^{2},
$$

which yields (3.4).

4. Approximation properties. We give approximation results that are extensions to tensors of results for the matrix case in [8].

4.1. Local quasi-optimality. If the low-rank approximation problem (1.1) has a continuously differentiable best approximation $\boldsymbol{X}(t) \in \mathcal{M}_{\mathbf{r}}$, then the error of (1.2) can be bounded in terms of the best-approximation error $\|\boldsymbol{X}(t)-\mathcal{A}(t)\|$. The result requires a bound on $\dot{\mathcal{A}}(t)$ :

$$
\|\dot{\mathcal{A}}(t)\| \leq \mu \quad \text { for } \quad 0 \leq t \leq \bar{t} .
$$

THEOREM 4.1. Suppose that a continuously differentiable best approximation $\boldsymbol{X}(t) \in \mathcal{M}_{\mathbf{r}}$ to $\mathcal{A}(t)$ exists for $0 \leq t \leq \bar{t}$. Let $\rho>0$ be such that the smallest nonzero singular value of the $n$th unfolding of $\boldsymbol{X}(t)$ satisfies $\sigma_{r_{n}}\left(\mathbf{X}_{(n)}(t)\right) \geq \rho$ for $n=1, \ldots, N$, and assume that the best-approximation error is bounded by $\|\boldsymbol{X}(t)-\mathcal{A}(t)\| \leq c \rho$ for $0 \leq t \leq \bar{t}$, with $c$ of Lemma 3.2. Then, the approximation error of the dynamical low-rank approximation (1.2) with initial value $\mathbf{y}(0)=\boldsymbol{X}(0)$ is bounded by

$$
\|\mathbf{y}(t)-\boldsymbol{X}(t)\| \leq 2 \beta e^{\beta t} \int_{0}^{t}\|\boldsymbol{X}(s)-\mathcal{A}(s)\| d s \quad \text { with } \beta=C \mu \rho^{-1}
$$

for $t \leq \bar{t}$ and as long as the right-hand side remains bounded by $c \rho$. Here, $C$ and $c$ are the constants of Lemma 3.2 .

Proof. With Lemma 3.2 at hand, the proof is essentially the same as that of Theorem 5.1 in [8]. The error of the best approximation $\boldsymbol{X}-\mathcal{A}$ is orthogonal to the tangent space $\mathcal{T}_{\boldsymbol{X}} \mathcal{M}_{\mathbf{r}}$, or equivalently, $\mathcal{P}(\boldsymbol{X})(\boldsymbol{X}-\mathcal{A})=\mathbf{0}$. We differentiate this relation with respect to $t$ and denote $\left(\mathcal{P}^{\prime}(\boldsymbol{X}) \cdot \mathcal{B}\right) \dot{\boldsymbol{X}}=\frac{d}{d t} \mathcal{P}(\boldsymbol{X}(t)) \mathcal{B}$ to obtain

$$
\mathcal{P}(\boldsymbol{X})(\dot{\boldsymbol{X}}-\dot{\mathcal{A}})+\left(\mathcal{P}^{\prime}(\boldsymbol{X}) \cdot(\boldsymbol{X}-\mathcal{A})\right) \dot{\boldsymbol{X}}=0 .
$$

Since $\dot{\boldsymbol{X}} \in \mathcal{T}_{\boldsymbol{X}} \mathcal{M}_{\mathbf{r}}$, we have $\mathcal{P}(\boldsymbol{X}) \dot{\boldsymbol{X}}=\dot{\boldsymbol{X}}$, and the equation becomes

$$
\left(\mathfrak{J}-\mathcal{P}^{\prime}(\boldsymbol{X}) \cdot(\boldsymbol{X}-\mathcal{A})\right) \dot{\boldsymbol{X}}=\mathcal{P}(\boldsymbol{X}) \dot{\mathcal{A}} .
$$

Lemma 3.2 and the condition $d:=\|\boldsymbol{X}-\mathcal{A}\| \leq c \rho$ yield

$$
\left\|\mathcal{P}^{\prime}(\boldsymbol{X}) \cdot(\boldsymbol{X}-\mathcal{A})\right\| \leq C \rho^{-1} d \leq C c \leq \frac{1}{2},
$$

and hence (4.2) can be solved for $\dot{X}$ to yield

$$
\dot{\boldsymbol{X}}=\mathcal{P}(\boldsymbol{X}) \dot{\mathcal{A}}+\mathcal{D} \quad \text { with } \quad\|\mathcal{D}\| \leq 2 C \rho^{-1} d \mu=2 \beta d .
$$


We subtract this equation from $(3.1)$, viz., $\dot{\boldsymbol{y}}=\mathcal{P}(\boldsymbol{y}) \dot{\mathcal{A}}$, and integrate from 0 to $t$. As long as $e(t):=\|\mathbf{y}(t)-\boldsymbol{X}(t)\| \leq c \rho$, Lemma 3.2 yields

$$
\|(\mathcal{P}(\mathbf{y})-\mathcal{P}(\boldsymbol{X})) \dot{\mathcal{A}}\| \leq C \rho^{-1} e \mu=\beta e,
$$

and hence we obtain

$$
e(t) \leq \beta \int_{0}^{t} e(s) d s+2 \beta \int_{0}^{t} d(s) d s .
$$

The result now follows with the Gronwall inequality.

4.2. A farther-reaching error bound. Smaller errors over longer time intervals are obtained if not only $\mathcal{X}-\mathcal{A}$, but also its derivative is small. We assume that $\mathcal{A}(t)$ is of the form

$$
\mathcal{A}(t)=\boldsymbol{X}(t)+\mathcal{E}(t), \quad 0 \leq t \leq \bar{t},
$$

where $\boldsymbol{X}(t) \in \mathcal{M}_{\mathbf{r}}$ (now this need not necessarily be the best approximation) with

$$
\|\dot{\mathfrak{X}}(t)\| \leq \mu
$$

and the derivative of the remainder term is bounded by

$$
\|\dot{\mathcal{E}}(t)\| \leq \varepsilon
$$

with a small $\varepsilon>0$.

THEOREM 4.2. In addition to the above assumptions, suppose that the smallest singular values of the unfoldings of $\boldsymbol{X}(t)$ are bounded from below by $\rho>0$. Then, the approximation error of (1.2) with initial value $Y(0)=X(0)$ is bounded by

$$
\|\mathbf{y}(t)-\boldsymbol{x}(t)\| \leq 2 t \varepsilon \quad \text { for } \quad t \leq \frac{\rho}{C \sqrt{\mu \varepsilon}}
$$

provided that $t \leq \frac{c \rho}{2 \varepsilon}$ and $t \leq \bar{t}$. The constants $C$ and $c$ are those of Lemma 3.2.

Proof. The proof follows that of Theorem 5.2 in [8]. We note $\dot{\boldsymbol{X}}=\mathcal{P}(\boldsymbol{X}) \dot{\boldsymbol{X}}$, rewrite (3.1) as $\dot{\boldsymbol{y}}=\mathcal{P}(\boldsymbol{y}) \dot{\boldsymbol{X}}+\mathcal{P}(\boldsymbol{y}) \dot{\mathcal{E}}$, and subtract the two equations. We observe

$$
(\mathcal{P}(\boldsymbol{y})-\mathcal{P}(\boldsymbol{x})) \dot{\boldsymbol{x}}=-\left(\mathcal{P}^{\perp}(\boldsymbol{y})-\mathcal{P}^{\perp}(\boldsymbol{x})\right) \dot{\boldsymbol{x}}=-\mathcal{P}^{\perp}(\boldsymbol{y}) \dot{\boldsymbol{x}}=-\mathcal{P}^{\perp}(\boldsymbol{y})^{2} \dot{\boldsymbol{x}}
$$

We take the inner product with $\boldsymbol{y}-\boldsymbol{x}$ to obtain

$$
\begin{aligned}
\langle\boldsymbol{y}-\boldsymbol{x},(\mathcal{P}(\boldsymbol{y})-\mathcal{P}(\boldsymbol{x})) \dot{\boldsymbol{x}}\rangle & =-\left\langle\boldsymbol{y}-\boldsymbol{x}, \mathcal{P}^{\perp}(\boldsymbol{y}) \dot{\boldsymbol{x}}\right\rangle=-\left\langle\mathcal{P}^{\perp}(\boldsymbol{y})(\boldsymbol{y}-\boldsymbol{x}), \mathcal{P}^{\perp}(\boldsymbol{y}) \dot{\boldsymbol{x}}\right\rangle \\
& =\left\langle\mathcal{P}^{\perp}(\boldsymbol{y})(\boldsymbol{y}-\boldsymbol{x}),(\mathcal{P}(\boldsymbol{y})-\mathcal{P}(\boldsymbol{x})) \dot{\boldsymbol{x}}\right\rangle .
\end{aligned}
$$

With Lemma 3.2 and (4.4), (4.5) this yields

$$
\begin{aligned}
\langle\boldsymbol{y}-\boldsymbol{x}, \dot{\boldsymbol{y}}-\dot{\boldsymbol{x}}\rangle & =\left\langle\mathcal{P}^{\perp}(\boldsymbol{y})(\boldsymbol{y}-\boldsymbol{x}),(\mathcal{P}(\boldsymbol{y})-\mathcal{P}(\boldsymbol{x})) \dot{\boldsymbol{x}}\right\rangle+\langle\boldsymbol{y}-\boldsymbol{x}, \mathcal{P}(\boldsymbol{y}) \dot{\boldsymbol{E}}\rangle \\
& \leq C^{2} \rho^{-2} \mu\|\boldsymbol{y}-\boldsymbol{x}\|^{3}+\|\boldsymbol{y}-\boldsymbol{x}\| \cdot \varepsilon
\end{aligned}
$$

and on the other hand we have

$$
\langle\boldsymbol{y}-\boldsymbol{x}, \dot{\boldsymbol{y}}-\dot{\boldsymbol{x}}\rangle=\frac{1}{2} \frac{d}{d t}\|\boldsymbol{y}-\boldsymbol{x}\|^{2}=\|\boldsymbol{y}-\boldsymbol{x}\| \frac{d}{d t}\|\boldsymbol{y}-\boldsymbol{x}\| .
$$


Taken together, we obtain for $e(t)=\|\mathbf{y}(t)-\boldsymbol{x}(t)\|$ the differential inequality

$$
\dot{e} \leq \gamma e^{2}+\varepsilon, \quad e(0)=0,
$$

with $\gamma=C^{2} \rho^{-2} \mu$. Hence, $e(t)$ is majorized by the solution of

$$
\dot{y}=\gamma y^{2}+\varepsilon, \quad y(0)=0,
$$

which equals $y(t)=\sqrt{\varepsilon / \gamma} \tan (t \sqrt{\gamma \varepsilon})$ and is bounded by $2 t \varepsilon$ for $t \sqrt{\gamma \varepsilon} \leq 1$. Lemma 3.2 remains applicable as long as $2 t \varepsilon \leq c \rho$.

4.3. Systems without gaps between the singular values. The results of the preceding subsections only give satisfactory error bounds when there is a gap in the distribution of the singular values of the unfoldings so that essential and inessential singular values are widely separated. We now consider a situation where such a gap need not exist. We make the assumptions of Theorem 4.2 and further that $\boldsymbol{X}(t) \in \mathcal{M}_{\mathbf{r}}$ with all singular values of all unfoldings greater than $\rho>0$ has a Tucker decomposition

$$
\boldsymbol{X}(t)=\overline{\mathfrak{S}}(t) \stackrel{N}{\mathrm{X}} \overline{\mathbf{U}}_{n}(t)
$$

such that the following bounds hold for $n=1, \ldots, N$ and $0 \leq t \leq \bar{t}$ :

$$
\left\|\overline{\mathbf{S}}_{(n)}^{\dagger} \dot{\overline{\mathbf{S}}}_{(n)}\right\| \leq \kappa, \quad\left\|\dot{\mathbf{U}}_{n}(t)\right\| \leq \nu .
$$

Under these conditions we can show an $O(\varepsilon)$ error over times $O(1)$ even with $\rho \sim \varepsilon$.

TheOREM 4.3. Under the conditions of Theorem 4.2 and with (4.6)-(4.7), the approximation error of (1.2) with initial value $\mathbf{y}(0)=\boldsymbol{X}(0)$ is bounded by

$$
\|\boldsymbol{y}(t)-\boldsymbol{x}(t)\| \leq 2 t \varepsilon \quad \text { for } \quad t \leq \frac{1}{\sqrt{c_{1} \kappa+c_{2} \nu}} \sqrt{\frac{\rho}{\varepsilon}}
$$

with constants $c_{1}$ and $c_{2}$ that depend only on the order $N$. This holds as long as the right-hand side remains bounded by $c \rho$ with $c$ of Lemma 3.2.

Proof. From the proof of Theorem 4.2 we have the equation

$$
\langle\boldsymbol{y}-\boldsymbol{x}, \dot{\boldsymbol{y}}-\dot{\boldsymbol{x}}\rangle=-\left\langle\mathcal{P}^{\perp}(\boldsymbol{y})(\boldsymbol{y}-\boldsymbol{x}), \mathcal{P}^{\perp}(\boldsymbol{y}) \dot{\boldsymbol{x}}\right\rangle+\langle\boldsymbol{y}-\boldsymbol{x}, \mathcal{P}(\boldsymbol{y}) \dot{\boldsymbol{\varepsilon}}\rangle .
$$

For $e(t)=\|\mathbf{y}(t)-\boldsymbol{X}(t)\| \leq c \rho$, the proof of Lemma 3.2 shows that there is a homotopy

$$
\mathbf{y}(t, \tau)=\boldsymbol{S}(t, \tau) \stackrel{N}{X} \mathbf{U}_{n}(t, \tau)
$$

with

$$
\boldsymbol{y}(t, 1)=\mathbf{y}(t), \quad \boldsymbol{y}(t, 0)=\boldsymbol{x}(t)
$$

for which the Tucker factors are bounded by

$$
\begin{aligned}
\left\|\frac{\partial \mathcal{S}}{\partial \tau}(t, \tau)\right\| & \leq 2 e(t) \\
\left\|\frac{\partial \mathbf{U}_{n}}{\partial \tau}(t, \tau) \mathbf{S}_{(n)}(t, \tau)\right\| & \leq 2 e(t) \\
\left\|\frac{\partial \mathbf{U}_{n}}{\partial \tau}(t, \tau)\right\| & \leq 4 e(t) \rho^{-1} \leq 4 c .
\end{aligned}
$$


We write

$$
\begin{aligned}
& \mathcal{P}^{\perp}(\boldsymbol{y}(t)) \dot{\boldsymbol{X}}(t)=\mathcal{P}^{\perp}(\boldsymbol{y}(t))\left(\dot{\boldsymbol{s}}(t, 0) \underset{n=1}{\stackrel{N}{X}} \mathbf{U}_{n}(t, 0)+\sum_{n=1}^{N} \boldsymbol{S}(t, 0) \times_{n} \dot{\mathbf{U}}_{n}(t, 0) \underset{k \neq n}{\mathbf{X}} \mathbf{U}_{k}(t, 0)\right. \\
& \left.-\dot{\mathcal{S}}(t, 0) \underset{n=1}{\mathrm{X}} \mathbf{U}_{n}(t, 1)+\sum_{n=1}^{N} \boldsymbol{S}(t, 1) \times_{n} \dot{\mathbf{U}}_{n}(t, 0) \underset{k \neq n}{\mathbf{X}} \mathbf{U}_{k}(t, 1)\right),
\end{aligned}
$$

noting that $\mathcal{P}^{\perp}(\boldsymbol{y}(t))$ annihilates the terms in the second line, since they are in $\mathcal{T}_{\boldsymbol{y}} \mathcal{M}_{\mathbf{r}}$. We have

$$
\begin{aligned}
& \left\|\dot{\boldsymbol{S}}(t, 0) \underset{n=1}{\stackrel{N}{X}} \mathbf{U}_{n}(t, 1)-\dot{\mathcal{S}}(t, 0) \underset{n=1}{\stackrel{N}{X}} \mathbf{U}_{n}(t, 0)\right\| \\
& =\left\|\int_{0}^{1} \frac{\partial}{\partial \tau}\left(\dot{\boldsymbol{S}}(t, 0) \underset{n=1}{\underset{X}{X}} \mathbf{U}_{n}(t, \tau)\right) \partial \tau\right\| \\
& \left.=\| \int_{0}^{1} \sum_{n=1}^{N} \dot{\boldsymbol{s}}(t, 0) \times_{n} \frac{\partial \mathbf{U}_{n}}{\partial \tau}(t, \tau) \underset{k \neq n}{\mathbf{X}} \mathbf{U}_{k}(t, \tau)\right) d \tau \| \\
& \leq \int_{0}^{1}\left\|\frac{\partial \mathbf{U}_{n}}{\partial \tau}(t, \tau) \dot{\mathbf{S}}_{(n)}(t, 0) \underset{k \neq n}{\bigotimes} \mathbf{U}_{k}(t, \tau)^{T}\right\| d \tau \\
& \leq \int_{0}^{1}\left\|\frac{\partial \mathbf{U}_{n}}{\partial \tau}(t, \tau) \mathbf{S}_{(n)}(t, 0)\right\| \cdot\left\|\mathbf{S}_{(n)}(t, 0)^{\dagger} \dot{\mathbf{S}}_{(n)}(t, 0)\right\| d \tau .
\end{aligned}
$$

Writing

$$
\frac{\partial \mathbf{U}_{n}}{\partial \tau}(t, \tau) \mathbf{S}_{(n)}(t, 0)=\frac{\partial \mathbf{U}_{n}}{\partial \tau}(t, \tau) \mathbf{S}_{(n)}(t, \tau)-\frac{\partial \mathbf{U}_{n}}{\partial \tau}(t, \tau) \int_{0}^{\tau} \frac{\partial \mathbf{S}_{(n)}}{\partial \sigma}(t, \sigma) d \sigma
$$

and using the bounds (4.7) and (4.9) thus yields

$$
\left\|\dot{\boldsymbol{S}}(t, 0) \underset{n=1}{\mathrm{X}} \mathbf{U}_{n}(t, 1)-\dot{\boldsymbol{s}}(t, 0) \underset{n=1}{\stackrel{N}{X}} \mathbf{U}_{n}(t, 0)\right\| \leq(2 e(t)+8 c e(t)) \kappa=\tilde{c}_{1} \kappa e(t)
$$

with $\tilde{c}_{1}=2+8 c$. In the same way, the remaining terms in the above expression for $\mathcal{P}^{\perp}(\boldsymbol{y}(t)) \dot{\boldsymbol{X}}(t)$ are bounded by $\tilde{c}_{2} \nu e(t)$, and hence we have

$$
\left\|\mathcal{P}^{\perp}(\boldsymbol{y}(t)) \dot{\boldsymbol{X}}(t)\right\| \leq\left(\tilde{c}_{1} \kappa+\tilde{c}_{2} \nu\right) e(t) .
$$

Using this inequality in (4.8) and also the second estimate of Lemma 3.2, we obtain the differential inequality, as long as $e(t) \leq c \rho$,

$$
\dot{e} \leq \gamma e^{2}+\varepsilon
$$

with $\gamma=\rho^{-1} C\left(\tilde{c}_{1} \kappa+\tilde{c}_{2} \nu\right)$. With $c_{1}=C \tilde{c}_{1}$ and $c_{2}=C \tilde{c}_{2}$, the result now follows as at the end of the proof of Theorem 4.2.

4.4. Low-rank approximation of tensor differential equations. For the low-rank approximation to a solution of the tensor differential equation

$$
\dot{\mathcal{A}}=F(\mathcal{A}),
$$

condition (1.2) is replaced, at every time $t$, by

$$
\dot{\boldsymbol{y}} \in \mathcal{T}_{\boldsymbol{y}} \mathcal{M}_{\mathbf{r}} \quad \text { such that } \quad\|\dot{\boldsymbol{y}}-F(\boldsymbol{y})\|=\min !
$$


Equivalently, condition (2.9) is replaced by the Galerkin condition

$$
\langle\dot{\boldsymbol{y}}-F(\boldsymbol{y}), \mathcal{V}\rangle=0 \quad \text { for all } \mathcal{V} \in \mathcal{T}_{\boldsymbol{y}} \mathcal{M}_{\mathbf{r}},
$$

and correspondingly, the expression $\dot{\mathcal{A}}$ is replaced by $F(\boldsymbol{y})$ for $\boldsymbol{y}=\boldsymbol{S} \mathbf{X}_{n=1}^{N} \mathbf{U}_{N} \in \mathcal{M}_{\mathbf{r}}$ in the differential equations (2.11)

Theorems 4.1-4.3 extend to the low-rank approximation of matrix differential equations (4.11). We assume that $F$ has a moderate bound along the approximations,

$$
\|F(\boldsymbol{X}(t))\| \leq \mu, \quad\|F(\boldsymbol{y}(t))\| \leq \mu \quad \text { for } 0 \leq t \leq \bar{t},
$$

and satisfies a one-sided Lipschitz condition: there is a real $\lambda$ (positive or negative or zero) such that

$$
\langle F(\boldsymbol{y})-F(\boldsymbol{X}), \boldsymbol{y}-\boldsymbol{X}\rangle \leq \lambda\|\boldsymbol{y}-\boldsymbol{X}\|^{2}
$$

for all matrices $\boldsymbol{X}, \boldsymbol{y} \in \mathcal{M}_{\mathbf{r}}$. We further assume that for the best approximation $\boldsymbol{X}(t)$,

$$
\|F(\boldsymbol{X}(t))-F(\mathcal{A}(t))\| \leq L\|\boldsymbol{X}(t)-\mathcal{A}(t)\| \quad \text { for } \quad 0 \leq t \leq \bar{t},
$$

which is in particular satisfied if $F$ is Lipschitz continuous with Lipschitz constant $L$. Furthermore, assume that the best-approximation error is bounded by $\|\boldsymbol{X}(t)-\mathcal{A}(t)\| \leq$ $c \rho$ for $0 \leq t \leq \bar{t}$, with $c$ of Lemma 3.2.

We then have the following extension of the quasi-optimality result of Theorem 4.1 .

THEOREM 4.4. Suppose that a continuously differentiable best approximation $\boldsymbol{X}(t) \in \mathcal{M}_{\mathbf{r}}$ to a solution $\mathcal{A}(t)$ of (4.11) exists for $0 \leq t \leq \bar{t}$, and assume the bounds (4.14)-(4.16). Let $\rho>0$ be such that the smallest nonzero singular value of the $n$th unfolding of $\boldsymbol{X}(t)$ satisfies $\sigma_{r_{n}}\left(\mathbf{X}_{(n)}(t)\right) \geq \rho$ for $n=1, \ldots, N$, and assume that the best-approximation error is bounded by $\|\boldsymbol{X}(t)-\mathcal{A}(t)\| \leq c \rho$ with $c$ of Lemma 3.2, for $0 \leq t \leq \bar{t}$. Then, the approximation error of (4.13) with initial value $\mathbf{y}(0)=\boldsymbol{X}(0)$ is bounded in the Frobenius norm by

$$
\|\boldsymbol{y}(t)-\boldsymbol{X}(t)\| \leq(2 \beta+L) e^{(2 \beta+\lambda) t} \int_{0}^{t}\|\boldsymbol{X}(s)-\mathcal{A}(s)\| d s \quad \text { with } \beta=C \mu \rho^{-1}
$$

for $t \leq \bar{t}$ and as long as the right-hand side is bounded by $c \rho$.

Proof. Equation (4.13) rewritten as in (3.1) reads

$$
\dot{\boldsymbol{y}}=\mathcal{P}(\boldsymbol{y}) F(\boldsymbol{y}) \text {. }
$$

As in the proof of Theorem 4.1, we have the equation

$$
\dot{\boldsymbol{X}}=\mathcal{P}(\boldsymbol{X}) F(\mathcal{A})+\mathcal{D} \quad \text { with } \quad\|\mathcal{D}\| \leq 2 \beta d
$$

for $d=\|\boldsymbol{X}-\mathcal{A}\|$. We subtract the two equations, write

$$
\begin{aligned}
\mathcal{P}(\boldsymbol{y}) F(\boldsymbol{y})-\mathcal{P}(\boldsymbol{X}) F(\mathcal{A})-\mathcal{D}= & (\mathcal{P}(\mathbf{y})-\mathcal{P}(\boldsymbol{X})) F(\boldsymbol{X})+\mathcal{P}(\boldsymbol{X})(F(\boldsymbol{X})-F(\mathcal{A})) \\
& +(F(\mathbf{y})-F(\boldsymbol{X}))-\mathcal{P}^{\perp}(\mathbf{y})(F(\mathbf{y})-F(\boldsymbol{X}))-\mathcal{D},
\end{aligned}
$$

and take the inner product with $\boldsymbol{y}-\boldsymbol{X}$. With Lemma 3.2 we obtain

$$
\begin{aligned}
\langle\dot{\boldsymbol{y}}-\dot{\boldsymbol{x}}, \boldsymbol{y}-\boldsymbol{x}\rangle \leq & \beta\|\boldsymbol{y}-\boldsymbol{X}\|^{2}+L d\|\boldsymbol{y}-\boldsymbol{X}\| \\
& +\lambda\|\boldsymbol{y}-\boldsymbol{X}\|^{2}+\beta\|\boldsymbol{y}-\boldsymbol{X}\|^{2}+2 \beta d\|\boldsymbol{y}-\boldsymbol{x}\| .
\end{aligned}
$$


For $e=\|\boldsymbol{y}-\boldsymbol{X}\|$ this gives the differential inequality

$$
\dot{e} \leq(2 \beta+\lambda) e+(2 \beta+L) d, \quad e(0)=0,
$$

which yields the result.

We refer to [12, Theorem 4.1] for a related quasi-optimality result in a situation of a linear differential equation with an unbounded operator.

In the differential equation analogue of Theorem 4.2 with the splitting (4.3), we start from the equations $\dot{\boldsymbol{y}}-\dot{\boldsymbol{x}}=\mathcal{P}(\boldsymbol{y}) F(\boldsymbol{y})-\mathcal{P}(\boldsymbol{x}) \dot{\boldsymbol{x}}$ and $\dot{\boldsymbol{X}}=F(\boldsymbol{A})-\dot{\mathcal{E}}$, yielding

$$
\begin{aligned}
\dot{\boldsymbol{y}}-\dot{\boldsymbol{x}}= & (\mathcal{P}(\boldsymbol{y})-\mathcal{P}(\boldsymbol{X})) \dot{\boldsymbol{X}}-\mathcal{P}^{\perp}(\boldsymbol{y})(F(\boldsymbol{y})-F(\boldsymbol{X})) \\
& +(F(\boldsymbol{y})-F(\boldsymbol{X}))+\mathcal{P}(\boldsymbol{y})(F(\boldsymbol{x})-F(\mathcal{A}))+\mathcal{P}(\boldsymbol{y}) \dot{\mathcal{E}},
\end{aligned}
$$

where we now take the inner product with $\boldsymbol{y}-\boldsymbol{X}$. If it is additionally assumed that $F$ has Lipschitz constant $L$, then this leads to the differential inequality

$$
\dot{e} \leq C \rho^{-1}(\beta+L) e^{2}+\lambda e+L d+\varepsilon, \quad e(0)=0 .
$$

With $\widehat{\gamma}=C \rho^{-1}(\beta+L)$ and $\widehat{\varepsilon}=\varepsilon+L \max _{0 \leq t \leq \bar{t}} d(t)$, and with $\varphi(x)=\left(e^{x}-1\right) / x$, this yields the error bound

$$
\|\boldsymbol{y}(t)-\boldsymbol{X}(t)\| \leq 2 t \varphi(\lambda t) \widehat{\varepsilon} \quad \text { for } \quad t \varphi(\lambda t) \leq \frac{1}{2}(\widehat{\gamma} \widehat{\varepsilon})^{-1 / 2}
$$

and as long as $t \leq \bar{t}$ and $2 t \varphi(\lambda t) \widehat{\varepsilon} \leq c \rho$.

Theorem 4.3 is similarly extended to tensor differential equations.

5. Numerical Experiment. To illustrate the theoretical error analysis, we compute the dynamical low rank approximation of a time-dependent tensor $\mathcal{A}(t) \in$ $\mathbb{R}^{15 \times 15 \times 15 \times 15}$, which is constructed to give control over the order of magnitude of the approximation error: With $U=\left(U_{1}, \ldots, U_{4}\right)$ a set of matrices in $\mathbb{R}^{15 \times 10}$ with orthonormal columns, $\mathcal{B}$ a random core tensor in $\mathbb{R}^{10 \times 10 \times 10 \times 10}$, and $\mathcal{C} \in \mathbb{R}^{15 \times 15 \times 15 \times 15}$ a random perturbation, the time-dependent data tensor is constructed as

$$
\mathcal{A}(t)=\exp (t) \mathcal{B} \times{ }_{1} U_{1} \times{ }_{2} \cdots \times_{4} U_{4}+\varepsilon(t+1+\sin (3 t)) \mathcal{C}, \quad t \in[0,1] .
$$

We compute the dynamical tensor approximation (2.9), where $\boldsymbol{y}$ is defined with a core tensor of dimension $10 \times 10 \times 10 \times 10$. We numerically solve the differential equations (2.11), taking as initial value an approximate best approximation computed by alternating least squares [1]. As time integrator, we use the implicit midpoint rule $\left(y_{n+1}=y_{n}+h f\left(\frac{1}{2}\left(y_{n}+y_{n+1}\right)\right)\right.$ for a differential equation $\left.y^{\prime}=f(y)\right)$ with step size $h=10^{-4}$, where the nonlinear equations in each step are solved by fixed point iteration with a stopping criterion in a norm combining the tensor norm for the core tensor and the Frobenius norms of the factor matrices. Figure 5 shows the approximation errors $\|\boldsymbol{y}(t)-\mathcal{A}(t)\|$ as functions of $t$ for $(5.1)$ with $\varepsilon \in\left\{10^{-5}, 10^{-4}, 10^{-3}, 10^{-2}, 10^{-1}\right\}$. We observe that indeed the error of the approximation is proportional to $\varepsilon$ and grows only moderately as a function of $t$ (note the logarithmic scaling of the vertical axis).

To demonstrate the potential computational advantage of dynamical tensor approximation versus pointwise approximation, we compare the computation time for our dynamical tensor approximation to pointwise approximation by alternating least squares as implemented in the MATLAB routines [1]. In the same setting as above, the tensor from (5.1) (with $\varepsilon=10^{-2}$ ) is approximated by a tensor with a core tensor of dimension $10 \times 10 \times 10 \times 10$. Figure 5 shows that the computation time is about 


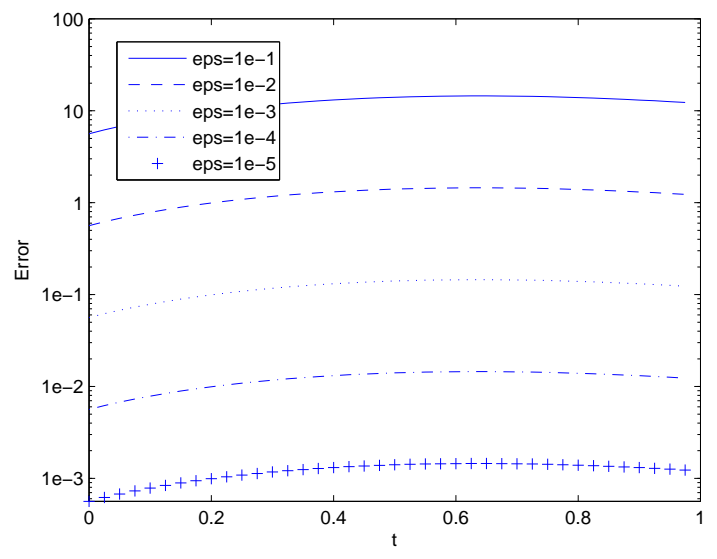

FIG. 5.1. Error of the approximation of rank $(10,10,10,10)$ for $\mathcal{A}$ from (5.1) with $\varepsilon \in$ $\left\{10^{-5}, 10^{-4}, 10^{-3}, 10^{-2}, 10^{-1}\right\}$. Interval $t \in[0,1]$, step-size $h=10^{-4}$.

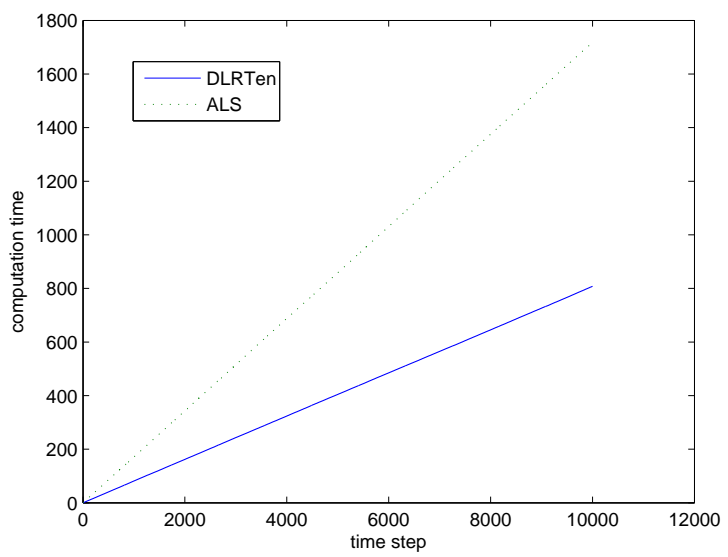

FiG. 5.2. Comparison of the computation times for Dynamical Low Rank Tensor Approximation (DLRTen) and pointwise approximation by Alternating Least Squares (ALS). Approximations of rank $(10,10,10,10)$ for $\mathcal{A}$ from (5.1) with $\varepsilon=10^{-2}$. Interval $t \in[0,1]$, step-size $h=10^{-4}$.

halved by adopting our approach. In other examples that we computed, this gain is larger when the size of the core tensor is smaller as compared to the original tensor size or when $\dot{\mathcal{A}}$ is significantly sparser than $\mathcal{A}$, as has similarly been observed in the dynamical low-rank approximation of large data matrices [15].

For further numerical examples and comparisons, we refer to [15], where in particular the dynamical low-rank approximation of the 3-tensors arising from the spatial discretization of a 3-dimensional reaction-diffusion PDE is considered.

Acknowledgment. We thank Tamara Kolda for helpful comments on a preliminary version and for useful hints to the literature.

This work was supported by the Austrian Academy of Sciences' APART program and by DFG, SPP 1324. 


\section{REFERENCES}

[1] B. W. Bader And T. G. KoldA, MATLAB Tensor Toolbox Version 2.3, 2009. http://csmr.ca.sandia.gov/ tgkolda/TensorToolbox/

[2] M. H. Beck, A. JÄCKLe, G. A. Worth, And H.-D. Meyer, The multiconfiguration timedependent Hartree (MCTDH) method: A highly efficient algorithm for propagating wavepackets, Phys. Rep., 324 (2000), pp. 1-105.

[3] V. DE SiLva AND L.-H. LiM, Tensor rank and the ill-posedness of the best low-rank approximation problem, SIAM J. Matrix Anal. Appl., 30 (2008), pp. 1084-1172.

[4] L. GRASEDYCK, Existence and computation of a low Kronecker-rank approximant to the solution of a tensor system with tensor right-hand side, Computing, 72 (2004), pp. 247-265.

[5] L. GrasedycK, Hierarchical singular value decomposition of tensors, Preprint 20, DFG SPP 1324, 2009. http://www.dfg-spp1324.de/download/preprints/preprint020.pdf

[6] W. HackBUsch And B. KhoromskiJ, Tensor-product approximation of operators and functions in high dimensions, J. Complexity, 23 (2007), pp. 137-168.

[7] R.A. Horn and C.R. Johnson, Matrix Analysis, Cambridge Univ. Press, Cambridge, 1985.

[8] O. Koch And C. Lubich, Dynamical low rank approximation, SIAM J. Matrix Anal. Appl., 29 (2007), pp. 434-454

[9] T. G. KoldA And B. W. BADER, Tensor decompositions and applications, Sandia Report SAND2007-6702, Sandia National Laboratories, Albuquerque, New Mexico 87185 and Livermore, California 94550, 2007.

[10] L. De Lathauwer, B. De Moor, And J. VAndewalle, A multilinear singular value decomposition, SIAM J. Matrix Anal. Appl., 21 (2000), pp. 1253-1278.

[11] - On the best rank-1 and rank- $\left(R_{1}, \ldots, R_{n}\right)$ approximation of higher-order tensors, SIAM J. Matrix Anal. Appl., 21 (2000), pp. 1324-1342.

[12] C. Lubich, On variational approximations in quantum molecular dynamics, Math. Comp., 74 (2005), pp. 765-779

[13] C. Lubich, From Quantum to Classical Molecular Dynamics: Reduced Models and Numerical Analysis, Zurich Lectures in Advanced Mathematics, Europ. Math. Soc., Zurich, 2008.

[14] H.-D. Meyer, F. Gatti, And G. A. Worth, eds., Multidimensional Quantum Dynamics: MCTDH Theory and Applications, Wiley-VCH, Weinheim, Berlin, 2009.

[15] A. Nonnenmacher And C. Lubich, Dynamical low rank approximation: Applications and numerical experiments, Math. Comput. Simulation, 79 (2009), pp. 1346-1357.

16] J. Sun, S. Papadimitriou, And P. S. Yu, Window-based tensor analysis on high-dimensional and multi-aspect streams, in ICDM '06: Proceedings of the Sixth International Conference on Data Mining, Washington, DC, USA, 2006, IEEE Computer Society, pp. 1076-1080.

[17] J. Sun, D. TAO, AND C. FAlOutsos, Beyond streams and graphs: dynamic tensor analysis, in KDD '06: Proceedings of the 12th ACM SIGKDD International Conference on Knowledge Discovery and Data Mining, New York, NY, USA, 2006, ACM, pp. 374-383.

[18] H. WANG AND M. THOSS, Multilayer formulation of the multiconfiguration time-dependent Hartree theory, J. Chem. Phys., 119 (2003), PP. 1289-1299.

[19] T. Zhang And G. Golub, Rank-one approximation to high order tensors, SIAM J. Matrix Anal. Appl., 23 (2001), pp. 534-550. 